\title{
Evaluation of Potential Employee Exposures to Mycobacterium tuberculosis in a Zoo's Elephant Exhibit
}

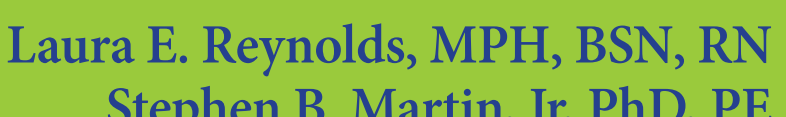

Stephen B. Martin, Jr, PhD, PE

R. Reid Harvey, DVM, MPH

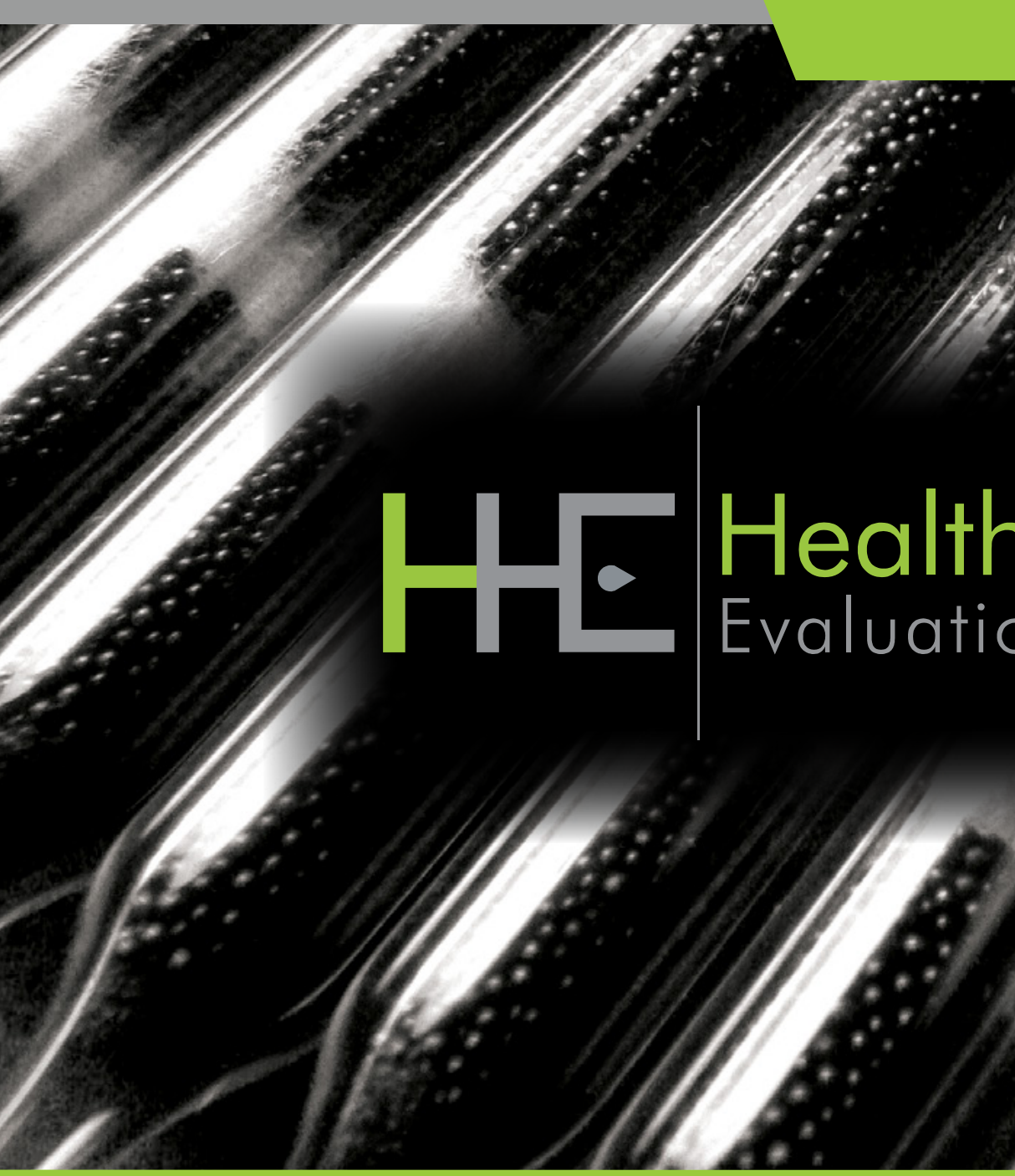

Report No. 2017-0096-3318

May 2018

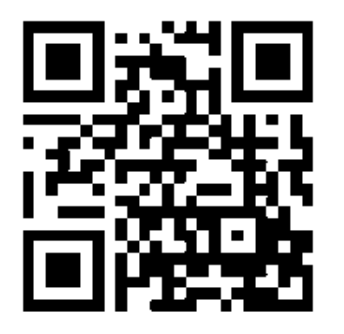

U.S. Department of Health and Human Services

Centers for Disease Control and Prevention

National Institute for Occupational Safety and Health

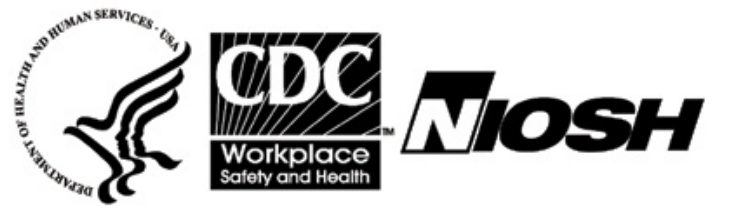




\section{Contents}

Highlights. i

Abbreviations ..................................... iv

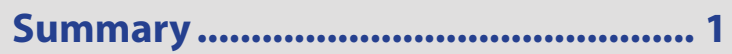

Introduction ...................................... 2

Background............................................. 2

Methods ............................................... 4

Results \& Discussion .............................. 6

Conclusions................................... 11

Recommendations........................... 11

References....................................... 15

Acknowledgements............................ 19

The employer is required to post a copy of this report for 30 days at or near the workplace(s) of affected employees. The employer must take steps to ensure that the posted report is not altered, defaced, or covered by other material.

The cover photo is a close-up image of sorbent tubes, which are used by the HHE Program to measure airborne exposures. This photo is an artistic representation that may not be related to this Health Hazard Evaluation. 


\section{Highlights of this Evaluation}

The Health Hazard Evaluation Program received a management request to determine the risk of zoo employees working in and around an elephant facility where four elephants were diagnosed with active tuberculosis disease over the last four years. NIOSH evaluated the ventilation systems, work practices, occupational health protocols, reviewed medical records and participated in a tuberculosis meeting.

\section{What We Did}

- We toured the elephant facility in May 2017.

- We interviewed zoo staff, observed work practices, evaluated the zoo's occupational health protocols, and assessed the ventilation systems in the elephant barn and indoor exhibit.

- We reviewed occupational health records provided by the zoo's occupational health provider.

- We participated in a Tuberculosis (TB) meeting hosted by the zoo at the request of the local health department in July 2017.

\section{What We Found}

- Record keeping for the Tuberculosis Monitoring Program and Respiratory Protection Program needed improvement.

- The Tuberculosis Exposure Control Plan needed improvement.

- The ventilation systems in the elephant barn and indoor exhibit were adequate.

\section{What the Employer Can Do}

- Designate a qualified person to ensure adequate record keeping for the Tuberculosis Monitoring

We evaluated the employees' work practices, occupational health protocols, reviewed occupational health records, assessed the ventilation systems in the elephant barn and indoor exhibit, and participated in a tuberculosis meeting hosted by the zoo at the request of the local health department. We recommended improvements with record keeping for the Tuberculosis Monitoring Program and Respiratory Protection Program and compliance with the Respiratory Protection Program. Program and the Respiratory Protection Program.

- Clearly define employee exposure classifications in the Tuberculosis Exposure Control Plan.

- Ensure employees adhere to the Respiratory Protection Program.

- Ensure standard procedures for personal protective equipment (PPE) are clearly established, communicated to employees, and enforced.

- Have N95 respirators available to employees and volunteers working with or around any elephant. 


\section{What Employees Can Do}

- Get a tuberculosis screening test at least every year if your last test was negative or you have not been tested.

- Get a clinical symptom screening for tuberculosis disease every year if you had a positive tuberculosis test in the past.

- Properly wear personal protective equipment listed in the zoo's occupational health protocols.

- Wear an N95 respirator while working with an elephant with active TB disease. 
This page left intentionally blank 


\section{Abbreviations}

$\begin{array}{ll}\text { ACH } & \text { Air changes per hour } \\ \text { CDC } & \text { Centers for Disease Control and Prevention } \\ \text { cfm } & \text { Cubic feet per minute } \\ \text { CFR } & \text { Code of Federal Regulations } \\ \text { EA } & \text { Exhaust air } \\ \text { HEPA } & \text { High efficiency particulate air } \\ \text { HRU } & \text { Heat recovery unit } \\ \text { HVAC } & \text { Heating, ventilation, and air-conditioning } \\ \text { IGRA } & \text { Interferon-Gamma Release Assays } \\ \text { MERV } & \text { Minimum efficiency reporting value } \\ \text { MDR } & \text { Multi-drug resistant } \\ \text { NIOSH } & \text { National Institute for Occupational Safety and Health } \\ \text { nm } & \text { Nanometers } \\ \text { OA } & \text { Outdoor air } \\ \text { OSHA } & \text { Occupational Safety and Health Administration } \\ \text { PAPR } & \text { Powered air-purifying respirator } \\ \text { PPE } & \text { Personal protective equipment } \\ \text { TAB } & \text { Testing and balancing } \\ \text { TB } & \text { Tuberculosis } \\ \text { TST } & \text { Tuberculin skin test } \\ \text { UV } & \text { Ultraviolet } \\ \text { UV-C } & \text { Ultraviolet-C (wavelengths between } 100 \mathrm{~nm} \text { and 280nm) } \\ \text { UVGI } & \text { Ultraviolet germicidal irradiation } \\ \end{array}$




\section{Summary}

In April 2017, the National Institute for Occupational Safety and Health received a health hazard evaluation request from the occupational health and safety manager at a zoo. The request stated concerns about employee exposures to elephants with active tuberculosis (TB) disease. In May 2017, we conducted a site visit and toured the elephant facility, interviewed employees who worked with elephants, observed work practices, evaluated the zoo's occupational health protocols, and assessed the ventilation systems in the elephant barn and indoor exhibit. We also reviewed occupational health records provided by the zoo's occupational health provider. We returned in July 2017 and participated in a TB meeting hosted by the zoo at the request of the local health department.

After reviewing the occupational health protocols, we determined that the zoo's Tuberculosis Exposure Plan needed improvement, specifically to clearly define employee exposure classifications. We also found that the Tuberculosis Monitoring Program and Respiratory Protection program needed better record keeping. We observed employees properly donning and doffing personal protective equipment. However, we also observed employees not wearing N95 respirators properly. We interviewed seven employees who worked with elephants. No employees voiced concerns about their health or working around elephants with TB disease. The ventilation systems assessment confirmed that the ventilation within the elephant barn and indoor exhibit were adequate.

We recommended that the zoo assign a designated person to ensure adequate record keeping for the Respiratory Protection Program and Tuberculosis Monitoring Program. We recommended to clearly define parts of the Tuberculosis Exposure Control Plan and ensure employees adhere to the Respiratory Protection Program. We also recommended that N95 respirators be available to any employee who works with the elephant herd. 


\section{Introduction}

The Health Hazard Evaluation Program received a request in April 2017 from the occupational health and safety manager about employee exposures to tuberculosis (TB) at a zoo. Since 2013, four Asian elephants at the zoo had been diagnosed with TB disease, most recently in April 2017. We visited the zoo in May 2017 and toured the elephant facility, assessed the ventilation systems (elephant barn and indoor exhibit), observed work practices, evaluated the zoo's occupational health protocols, and conducted informal, confidential interviews with elephant keepers. We sent an interim letter to the zoo, state health department, local health department, OSHA Region X office, and an employee representative which contained interim engineering, administrative, and personal protective equipment (PPE) recommendations. We also reviewed occupational health records obtained from the zoo's occupational health provider. In July 2017, we participated in a TB meeting at the request of the zoo and local health department. The purpose of the meeting was to discuss the local health department's recommendations for the zoo.

\section{Background}

\section{Tuberculosis}

TB is a disease caused by Mycobacterium tuberculosis bacteria. Although generally found in the lungs, TB bacteria can attack any part of the body. An animal or person can inhale TB bacteria which can begin to grow and move to other places in the body. An individual becomes infected with TB when the bacteria begin to grow, but the individual does not necessarily become sick. When this happens in humans, it is called latent TB infection, or LTBI. Individuals with LTBI do not have symptoms and cannot spread TB to others, but are at risk for developing TB disease. People with LTBI can take medicine to decrease the likelihood of developing TB disease in the future. Without treatment, 5\%-10\% [Division of Tuberculosis Elimination 2014] of individuals infected with TB will develop TB disease in their lifetime. TB disease causes the person to become sick and able to spread TB bacteria to others, and occurs when the TB bacteria actively grow in the body and overcome the immune system. Therefore, individuals with weakened immune systems are more likely to develop TB disease. Generally, TB bacteria are spread through close, prolonged contact with a person or animal with TB disease.

\section{Elephant TB}

Reports of TB-like illnesses in Asian elephants have been documented for thousands of years [Chalke 1962] and the point prevalence of TB among captive Asian elephants in the United States from 1997-2011 was estimated to be 5.1\% and 0 for African elephants [Feldman et al. 2013]. Transmission of $M$. tuberculosis has been documented between elephants and humans [Michalak et al. 1998]. The local health department with jurisdiction where this zoo is geographically located also found evidence of $M$. tuberculosis exposure between elephants and employees with close contact with the elephants [Zlot et al. 2016].

Since 2015, the United States Department of Agriculture (USDA), Division of Animal 
and Plant Health Inspection Services (APHIS), no longer regulates the surveillance, diagnosis and treatment of TB in elephants within the U.S. That responsibility now rests with the lead veterinarian treating the elephant with TB. However, the Stakeholders Task Force on Management \& Research Priorities of Tuberculosis in Elephants published the Recommendations for the Diagnosis, Treatment and Management of Tuberculosis in Elephants in Human Care in 2017. This detailed document gives recommendations for diagnostic testing, treatment, and monitoring of the elephant and herd during treatment, as well as human health considerations [Stakeholders Task Force on Management \& Research Priorities of Tuberculosis in Elephants 2017].

TB in elephants is usually diagnosed by trunk wash culture and elephants in captivity should be tested at least annually. Routine trunk wash culture consists of a series of three trunk wash samples collected on separate days within a one-week period [Stakeholders Task Force on Management \& Research Priorities of Tuberculosis in Elephants 2017]. Culture is the only definitive method to diagnose active TB disease and although trunk wash culture can lack sensitivity, it is still considered the gold-standard for diagnosing for TB in elephants. Other ancillary tests such as Dual Path Platform ${ }^{\mathrm{TM}}$ (DPP) and MultiAntigen Print ImmunoAssay ${ }^{\mathrm{TM}}$ (MAPIA) can be used in conjunction with trunk wash cultures. The DPP is a rapid test to detect antibodies of $M$. tuberculosis in blood, while the MAPIA uses a panel of 12 defined antigens to identify active disease. At the time of our visit, the elephant with active disease received trunk wash cultures weekly, while the rest of the herd received trunk wash cultures monthly. The zoo also performed DPP on all of the elephants monthly, and if positive, a MAPIA test was completed. Because diagnosing and treating elephants with TB can be difficult, time-consuming, and expensive, more research is needed to better understand the science of tuberculosis in elephants, risk to humans, and to develop improved screening methods for TB in elephants.

\section{TB Transmission at the Zoo}

In the summer of 2013, Elephant A was diagnosed with TB on a routine annual trunk wash culture. Due to the concern for potential zoonotic transmission of TB from elephants to humans, the local health department conducted a contact investigation [Zlot et al. 2016]. Nineteen zoo employees were considered close contacts to the elephant and six converted to positive on tuberculin skin tests (TST). During Elephant A's contact investigation, the zoo increased trunk wash cultures to monthly for the rest of the herd and in the fall of 2013, Elephant B tested positive on trunk wash culture and was diagnosed with TB. The same 19 employees who were close contacts with Elephant A were also close contacts with Elephant B.

Due to the number of positive TST conversions among close contacts, the local health department expanded their investigation, but did not find any other individuals who converted. Additionally, in the fall of 2013, an adjacent local health department discovered that a 2012 pleural TB case was a volunteer at the zoo prior to his/her diagnosis. Through genotype analysis, this zoo volunteer's cultured isolate was found to be almost identical to Elephant A and Elephant B, suggesting transmission. 
In the spring of 2014, Elephant $C$ tested positive on trunk wash culture for TB. All three elephants $(\mathrm{A}-\mathrm{C})$ began TB treatment at the time of diagnosis. Elephant A and Elephant $\mathrm{C}$ were euthanized in 2015 for reasons other than TB before they finished TB treatment. Elephant B was unable to tolerate treatment and developed multi-drug resistant (MDR) TB and was euthanized in February 2017 after being unable to tolerate MDR treatment.

In April 2017, Elephant D was diagnosed with TB by trunk wash culture. Since Elephant A's diagnosis in 2013, zoo employees with close elephant herd contact received TB testing every six months and if they had a history of a positive TB test, TB symptom screening once a year. One additional employee converted to positive on TST in 2016, which was considered to be within the expected range of conversions. Although other species at the zoo can become infected with M. tuberculosis, no other animals have tested positive through the zoo's routine testing schedules. To determine the extent of risk to employees working with and around the elephants and provide recommendations, zoo management requested a Health Hazard Evaluation from NIOSH.

\section{Methods}

Our objectives in this evaluation were the following:

1. Observe workplace practices when working with elephants

2. Evaluate the zoo's employee health protocols related to tuberculosis

3. Assess the ventilation systems in the elephant barn and indoor exhibit

4. Conduct informal, confidential interviews with elephant keepers

5. Review occupational health records from the zoo's occupational health provider

\section{May 2017 Site Visit}

We visited the zoo in May 2017. On May 31, 2017, we held an opening meeting with the occupational safety and health manager and met with the elephant curator (employee representative). At the end of our visit, we held a closing meeting with the occupational health and safety manager, zoo director, assistant zoo director, lead veterinarian, elephant curator, and local health department official.

\section{Facility Tour}

We toured the zoo, specifically the veterinary facility and the elephant facility. This zoo has an Asian elephant facility which includes an outdoor exhibit, indoor exhibit, and elephant barn (which included elephant stalls, an isolation stall, worker areas, and elephant food storage area). We assessed the ventilation systems in the indoor exhibit and barn (including the isolation stall and worker area). We observed work practices and the donning and doffing of PPE. 


\section{Review of the Zoo's Written Protocols}

We reviewed four written occupational health protocols related to TB exposure and PPE. The elephant facility had a TB Elephant and Staff Care Protocol specific to working with elephants. The zoo had two occupational health plans related to TB exposure: Tuberculosis Exposure Control Plan and N95 Respirator Plan. The municipality that manages the zoo also had a Respiratory Protection Program which all facilities they manage were to follow. We also reviewed available TB testing results and Respiratory Protection Program records, as well as relevant training records.

\section{Ventilation Assessment}

We visually assessed the three heat recovery units (HRUs) serving the barn and indoor exhibit. The heat pump system serving the elephant barn food storage area and worker office areas in the barn were also assessed. Air flow measurements were not taken during the visit. However, we met with the zoo's building automation manager and reviewed the real-time performance of each of the four key mechanical systems in the zoo's building automation system. We reviewed the sequence of operations for the three HRUs. We also reviewed a thorough testing and balancing (TAB) report for all elephant exhibit building systems provided to the zoo by Neudorfer Engineers, Inc. (Portland, OR). The data presented in the TAB report were collected during April and May 2015 and represent the final adjustments to the ventilation systems after installation and commissioning. In key areas of the elephant barn, ventilation smoke tubes were used to describe the direction of airflow between adjacent spaces.

\section{Informal, Confidential Interviews}

We invited all elephant keepers working during our initial visit in May 2017 to participate in informal, confidential interviews. During these voluntary interviews, we discussed their knowledge about TB disease, their known exposures, and asked if they had any related health concerns. We also educated employees about TB disease.

\section{Employee Occupational Health Record Review}

We requested and reviewed occupational health medical records from the zoo's occupational health provider in accordance with the Code of Federal Regulations (45 CFR § 164.512(b) (1) (i)), Federal Privacy Act (5 U.S.C. § 552a,), and the OSH Act (United States v. Amalgamated Life Insurance Company, 534 F. Supp. 676 (S.D.N.Y. 1982)).

\section{July 2017 Tuberculosis Meeting}

We returned July 25-26, 2017 at the request of the local health department. The zoo, in coordination with the local health department, hosted a TB meeting in which experts in tuberculosis, public health, occupational health, and veterinary medicine participated. The meetings' objectives were to assess the risk of tuberculosis to the general public, staff, and volunteers, and determine ways to improve the understanding of epidemiology and prevention of TB transmission between people and elephants. 


\section{Results and Discussion}

\section{Facility Tour and General Observations}

The zoo's elephant facility expanded and the new barn and indoor and outdoor exhibits opened in 2015. This new elephant facility covered more than 6 acres and included an outside habitat, indoor exhibit, and elephant barn. The barn had multiple stalls (including an isolation stall) and an elephant food storage area on the first floor. The second floor had office space for elephant keepers and a catwalk where you could oversee the barn.

At the time of our visit in May 2017, Elephant D lived in the elephant barn, not the isolation stall. When Elephant D was diagnosed in April 2017, the isolation stall was awaiting a TB mitigation cleanup since the previous elephant housed in the isolation stall had MDR TB. The new elephant facility had adequate space to keep Elephant D in the elephant barn, removed from the rest of the herd. Elephant D had access to the outdoors at specific times during the day, but not at the same times as the rest of the herd. The other elephants were housed in the outdoor and indoor exhibits. If another elephant needed to go into a space occupied by Elephant D, the other elephant could do so at least two hours after Elephant D had vacated the area.

Although Elephant D and the rest of the current herd were separated since the time of Elephant D's TB diagnosis, it is likely the remainder of the herd was exposed to TB by Elephant D before separation and subsequent isolation. It takes a minimum of eight weeks for trunk wash cultures to grow TB and for the zoo to be notified of the results. During the lag time between the trunk wash culture being submitted and awaiting results, Elephant D lived and interacted with the remainder of the herd and they were likely exposed to TB. It is also likely that Elephant D was exposed to TB from Elephants A-C during this same lag time in the past. Similarly, workers could have been exposed during this lag time since they did not wear PPE while working with undiagnosed elephants.

The zoo had nine full-time employees primarily working with elephants (elephant keepers) and one employee who worked with elephants part-time, but usually worked with the rhinoceroses. The general public, zoo volunteers, and tours were prohibited in the elephant barn since Elephant A was diagnosed in 2013. When working directly with Elephant D, keepers wore N95 respirators, suits (such as Tyvek), boots, gloves, and protective eyewear in accordance with the zoo's TB Exposure Control Plan. One keeper was assigned to work with Elephant D each day and the schedule rotated. If a worker was in the elephant barn, but on the second floor, the workers wore N95 respirators only. No PPE was mandated when working with the rest of the herd. Per the zoo's protocols, activities such as power-washing (an aerosolizing procedure) in the elephant facility were prohibited.

\section{Review of Written Protocols and Observations}

\section{Tuberculosis Exposure Control Plan}

The TB Exposure Control Plan was written for the entire zoo and not specific to the elephant facility and keepers. This plan had eight sections: Employee exposure classifications, 
notification of TB hazard, Employee TB Monitoring Program, Exposure incident reporting, Procedures to isolate and manage TB infected animal care, Engineering controls, Personal protective equipment, and Exposure control plan review and update.

The Employee Exposure Classification section of the TB Exposure Control Plan identified three groups of zoo workers at risk for TB based on their type of exposure:

1. Close contacts (have direct access indoors to or are within 20ft (outdoors) of a TB infected animal at least one day a week)

2. Job type (animal health, animal care, animal nutrition and animal life support)

3. Any other employee who works in animal areas at least one day a week

The Employee TB monitoring program section of the TB Exposure Control Plan details how and when TB screening is required for the three groups listed above. Group 1 (close contacts) were required to be screened for TB upon hire and then twice yearly. Groups 2 and 3 were required to have TB screening annually, but only Group 2 was required to have a TB screening test upon hire. Per the plan, all Groups received TB screening if they transferred to another section not requiring screening or when they stopped working at the zoo. If a worker had a history of a positive TB screening test, they were required to obtain a symptom screening and evaluation from a physician annually. Workers received TB screening through the zoo's occupational health provider (a local hospital system).

The TB screening results were tracked in a Microsoft Excel ${ }^{\mathrm{TM}}$ spreadsheet. The spreadsheet included the work area for each employee, but the work area did not correlate with the employee exposure classifications in the TB Exposure Control Plan. The spreadsheet included dates the workers were screened, but did not have the results. Also, although workers with prior positive screening tests were highlighted in red, their annual symptom screenings and evaluation dates were not tracked or recorded. The tracking spreadsheet also did not specify the reason each employee was screened (e.g. annual vs. contact investigation). Therefore, we were unable to differentiate which employees received TB screening tests because they were exposed to elephants with active TB and those who received an annual TB screening test. As a result, the spreadsheet could not be used for adequate TB surveillance for zoo employees.

The PPE section of the TB exposure control plan stated that employees working directly with an animal with active TB were required to wear an N95 respirator (or higher), protective suits (such as Tyvek), gloves, boots, and a protective face shield. An N95 respirator (or higher) was required in known or suspected TB contaminated areas and while cleaning potentially contaminated surfaces. We observed workers wearing the required PPE per the plan and donning and doffing properly.

N95 Respirator Plan and Respiratory Protection Program

The zoo's N95 Respirator Plan and municipality's Respiratory Protection Program met the OSHA standards (29 CFR 1910.134) [Occupational Safety and Health Administration]. 
These protocols required documented annual training and fit-testing of respirators and described proper wear of the respirator. All elephant keepers were fit-tested to wear an N95 respirator upon hire. However, workers were not fit-tested annually and their respiratory protection training was not documented or available for review. We observed one employee make frequent adjustments to the respirator by moving it away from his/her face and three employees with facial hair wearing N95 respirators.

\section{Ventilation Assessment}

The HRUs associated with the isolation stall (HRU-1), elephant barn (HRU-2) and indoor exhibit (HRU-3) were in excellent condition. The three HRUs move air through the elephant enclosures in a single-pass fashion. All return air is exhausted outdoors; there was no recirculated air through the HRUs.

All three HRUs are designed in the same way and have similar modes of operation. Aside from being powered off, the HRUs can all operate in Normal (Occupied) Mode or Natural Ventilation (Exhaust Only) Mode. Other operating modes exist, but they are associated with unoccupied conditions and will not be addressed here.

In Normal Mode, outside air (OA) brought in through an OA damper passes through a filter bank with prefilters having a Minimum Efficiency Reporting Values (MERV) rating of 8 and then more efficient MERV 13 bag filters. This combination of filters would be effective at filtering out most droplet nuclei that cause TB, but they are only filtering air pulled in from outdoors; no recirculated air from the indoor spaces pass through these filters. The OA then flows through one side of an air-to-air heat exchanger and a hot water coil before it reaches the supply fan and is pushed into the occupied spaces. At the same time, exhaust air (EA) pulled from the occupied spaces pass through a relief fan and is then pushed through a MERV 8 filter and the other side of the air-to-air heat exchanger before being released outdoors through the EA damper. The air-to-air heat exchanger simply serves to save energy as heat from the EA is used to preheat OA when conditions allow; the air streams themselves do not mix. In Normal Mode, the supply and relief fans run continuously. HRU-1 in the isolation stall is set up so that 8,000 cubic feet per minute $(\mathrm{cfm})$ of EA is pulled from the stall while only $6,000 \mathrm{cfm}$ of OA is supplied to the space. This offset of $-2,000 \mathrm{cfm}$ is designed to provide 6 air changes per hour $(\mathrm{ACH})$ of outdoor air through the stall while maintaining the space under constant negative pressure to prevent the escape of any infection droplet nuclei. HRU-2 serving the elephant barn is set to supply 24,000 cfm of OA to the space while removing 23,000 cfm of EA (offset of $+1,000 \mathrm{cfm}$ ). These settings provide the stalls with $4 \mathrm{ACH}$ of outdoor air while maintaining the space under slightly positive pressure. HRU-3 serving the indoor exhibit is set to supply and exhaust 35,000 cfm (offset of $0 \mathrm{cfm}$ ) so that the space is maintained under neutral pressure.

When outdoor weather conditions are close to the desired indoor conditions, the three HRUs can be operated in Natural Ventilation Mode. Here, the OA damper, OA filter bank, air-toair heat exchanger, hot water coil, and supply fan are bypassed (the supply fan is powered off). On the EA side, the EA filter bank, air-to-air heat exchanger, and EA damper is also bypassed. However, the relief fan continues to operate at its normal flowrate setpoint. 
Here, OA is brought directly into the space by way of a natural ventilation damper. The air then flows through the relief fan and directly back outdoors through an EA bypass damper. Operating in this mode can provide similar air exchange rates and pressure relationships while saving energy. During our visit to the zoo in May 2017, HRU-1 serving the unoccupied isolation stall was operating in Natural Ventilation Mode, while HRU-2 and HRU-3 were both operating in Normal Mode.

When the HRUs are operated in Normal Mode, all air exhausted from the HRUs pass through a MERV 8 filter prior to being released directly outdoors. These exhaust filters are in place to protect the heat-recovery air exchanger from becoming fouled. Some employees mentioned safety concerns regarding changing the MERV 8 filters in the exhaust air stream because of the potential for them to be contaminated with Mycobacterium tuberculosis. Laboratory studies indicate that re-aerosolization of viable mycobacteria from filter material is not probable under normal conditions [Ko et al. 1998; Qian et al. 1997; Reponen et al. 1999]. However, the risks associated with handling filters with mycobacteria in ventilation systems under field-use conditions have not been evaluated. The Centers for Disease Control and Prevention (CDC) recommends that workers performing maintenance and replacing filters on any ventilation system with potential for M. tuberculosis contamination wear a respirator, eye protection, and gloves[Jensen et al. 2005]. When feasible, filters can be disinfected by spraying them with a $10 \%$ bleach solution or other appropriate mycobacteriacide before removal [Rutala et al. 1991].

During our May 2017 visit, testing with ventilation smoke showed that air from the elephant barn can pass around the closed doors into the second floor staff offices. This was not the case around the doors into the kitchen area on the first floor, because those doors had been previously sealed with tape and were inoperable. Installing better seals and door sweeps on the doors from the stall into the staff offices would likely help alleviate the likelihood of contaminated air from the elephant barn migrating into staff areas. Smoke testing also showed that air from the stall can pass under the second floor door into the corner of the indoor exhibit. However, the exhaust grilles for HRU-3 serving the indoor exhibit are just inside that door, so any infectious droplet nuclei passing under the door into the indoor exhibit should immediately be exhausted from the space.

In addition to assessing ventilation system, we had a discussion with staff regarding the possible use of ultraviolet germicidal irradiation (UVGI) systems in the indoor spaces of the elephant exhibit. UVGI is the use of UV energy to inactivate viral, bacterial, and fungal organisms so they are unable to replicate and potentially cause disease. The entire UV spectrum is capable of inactivating microorganisms, but UV-C energy provides the most germicidal effect, with 265 nanometers $(\mathrm{nm})$ being the optimum wavelength. Since solar UV-C energy is unavailable on the surface of the earth, UV-C radiation must be produced by artificial sources. A variety of available lamps produce some energy in the ultraviolet spectrum, but the majority of modern UVGI lamps create UV-C energy with an electrical discharge through a low-pressure gas (including mercury vapor) enclosed in a quartz tube. Roughly $95 \%$ of the energy produced by these lamps is radiated at a near-optimal wavelength of $253.7 \mathrm{~nm}$. 
Upper-air UVGI can be very effective at reducing airborne infectious aerosols in certain settings, especially those with recycled air and good air mixing. However, it is unlikely the technology would provide benefits in the indoor elephant areas given that they use singlepass ventilation and are well over two stories tall. Further, the use of UV energy to help disinfect infectious organisms in the sand on the floors of the indoor spaces is not warranted. UV-C energy can be effective at disinfecting non-porous surfaces, but will not penetrate sand to provide any appreciable disinfection levels.

\section{Informal, Confidential Interviews}

We interviewed all seven elephant keepers who were working during our initial site visit. Although each keeper reported he/she understood their risk of working with and being exposed to elephants with TB, three workers wanted more information about the risk of getting TB from surfaces and five workers asked if suits and boots were needed as PPE. We told them that TB is generally a respiratory droplet disease, therefore wearing an N95 respirator was very important and that surfaces around elephants with active TB were not likely to be a risk. No keeper could recall when they were last fit-tested for the N95 respirator. All keepers reported receiving a TB screening test or clinical symptom screen due to Elephant D's recent contact investigation. No other concerns were expressed.

\section{Occupational Medical Records Review}

We requested occupational medical records associated with TB screening from the zoo's occupational health provider. All zoo employees and volunteers were required to have annual TB screening to work at the zoo. Testing frequency increased for some workers due to the elephants being diagnosed with TB and their respective contact investigations. We reviewed medical records from 2013-2017 obtained from the occupational health provider. During each elephant contact investigation, the zoo required different groups of employees to be screened for TB as defined by their TB Exposure Control Plan. For example, during one investigation, all zoo employees were tested for $\mathrm{TB}$, while during a different investigation, only close contacts were tested. The TB testing form used by the occupational health provider for all TB tests included the employee's name and place of employment (zoo), but work area, job title, and employee exposure classification (from the TB Exposure Control Plan) were not included. Therefore, we were unable to review and organize the workers' testing results by exposures for surveillance. From 2013-2016, the occupational health provider tested workers using the TST. Because TSTs require a placement visit and another visit 48-72 hours later for it to be read, in the fall of 2016 the occupational health provider began using QuantiFERON TB (QFT) Gold tests on all zoo workers at management's request. A QFT is one blood draw and does not require a follow-up appointment (unless positive).

\section{July 2017 TB Meeting}

In July 2017, the zoo, in collaboration with the local health department, hosted a TB meeting to review and discuss the TB outbreak among elephants. The purpose of the meeting was for the local health department to gather information on the risk to the public, risk to employees, elephant TB epidemiology, and TB transmission prevention between elephants and humans. After the meeting, the local health department gave the zoo recommendations moving forward. Representatives from NIOSH, CDC, and the state and local health departments, 
as well as other experts in human and elephant TB attended the two-day meeting. At the meeting, NIOSH presented the interim recommendations from the HHE.

\section{Conclusions}

A health hazard to employees from exposure to M. tuberculosis existed at this zoo. The TB Exposure Control Plan, record-keeping for the TB Monitoring Program and Respiratory Protection Program, and adherence to the Respiratory Protection Program needed improvement. Our evaluation identified deficiencies in administrative controls and PPE use. The engineering controls in the new elephant barn and indoor exhibit were adequate.

\section{Recommendations}

On the basis of our findings, we recommend the actions listed below to reduce the risk of $M$. tuberculosis transmission from elephants to workers. We encourage the zoo to use a labormanagement health and safety committee or working group to discuss our recommendations and develop an action plan. Those involved in the work can best set priorities and assess the feasibility of our recommendations for the specific situation at the zoo.

Our recommendations are based on an approach known as the hierarchy of controls. This approach groups actions by their likely effectiveness in reducing or removing hazards. Unlike most occupational exposures, for TB, environmental controls, such as engineering controls, are considered a second line defense in the TB infection control program after administrative controls.

\section{Elimination and Substitution}

Eliminating or substituting hazardous processes or materials reduces hazards and protects employees more effectively than other approaches. Prevention through design, considering elimination or substitution when designing or developing a project, reduces the need for additional controls in the future.

1. In previous literature, euthanasia was considered for elephants that were poor candidates for treatment and showing clinical symptoms, or based on other factors according to the veterinarian's clinical discretion [United States Animal Health Association (USAHA) Elephant Tuberculosis Submcommittee 2012]. Euthanizing an elephant with active TB would decrease the likelihood of transmitting $M$. tuberculosis to workers. However, at this zoo, euthanasia was not considered an option unless no others were available, as with Elephant B which could not tolerate MDR treatment. Treatment of elephants with TB disease remains the preferred option.

\section{Administrative Controls}

The term administrative controls refers to employer-dictated work practices and policies to reduce or prevent hazardous exposures. Their effectiveness depends on employer commitment and employee acceptance. Regular monitoring and reinforcement are necessary 
to ensure that policies and procedures are followed consistently.

1. Another option is to substitute or change the treatment protocol. Although an LTBI diagnosis is not recognized in elephants, all elephants in this herd have likely been exposed to other elephants with TB, are potentially infected, and may develop active TB in the future. Treating all elephants in this herd with prophylactic TB medication could decrease the risk of elephants transmitting TB to workers. However, this change in treatment protocol would be expensive and has its own risks to the elephants (medication intolerance and developing MDR TB). Given the history of TB in this herd, we recommend treating and isolating any elephant in this herd with any positive TB screening test result, including DPP, not just a positive trunk wash culture. At this time $50 \%$ of the original elephant herd from 2013 has been diagnosed with TB disease, which is much higher than the estimated prevalence of 5.1\% among captive Asian elephants [Feldman et al. 2013]. The remaining elephants in the herd have had exposure to elephants with TB and quicker treatment and isolation of elephants with a positive TB test may decrease the risk to workers and other elephants.

2. Ensure employees understand potential hazards (e.g., TB) in the workplace and how to protect themselves. OSHA's Hazard Communication Standard, also known as the "Right to Know Law" (29 CFR 1910.1200) [Occupational Safety and Health Administration] requires that employees are informed and trained on potential work hazards and associated safe practices, procedures, and protective measures.

3. Continue TB training during working hours upon hire and then annually to ensure questions can be addressed, employees have an understanding of TB disease, symptoms, transmission and how to prevent it. Document these trainings in employee records. Information on these topics can be found on the CDC TB website at https:// www.cdc.gov/tb/.

4. Continue TB testing (TST or QFT) at least annually and upon hire for employees with a previously negative test. Ensure prompt medical evaluation if an employee's TB test is newly positive.

5. Ensure employees with a history of LTBI have a clinical evaluation and symptom screen for TB disease annually.

6. Ensure there is an adequate confidential record keeping system in place to track initial and follow-up TB screenings in the TB Monitoring Program. This should include a tracking system which includes why the worker was tested, employee exposure classifications, results, annual clinical evaluations, and information on required followup of positive tests. Designate a qualified person with a back-up to keep the tracking system up to date.

7. Treat all elephants with active TB disease or any positive TB test result and move them to the isolation stall as soon as possible. Keep the elephant in isolation until the attending veterinarian deems the elephant able to rejoin the herd.

8. Do not eat or smoke in the elephant enclosure areas. Always wash hands after working in the elephant enclosure areas and before eating or smoking. 


\section{Environmental Controls}

Environmental controls, such as engineering controls, reduce employees' exposures by removing the hazard from the process or by placing a barrier between the hazard and the employee. Engineering controls protect employees effectively without placing primary responsibility of implementation on the employee.

1. In the main elephant barn, install better seals and door sweeps on the doors from the stall into the staff offices (and kitchen area if the tape is removed from those doors).

2. While laboratory studies indicate that re-aerosolization of viable mycobacteria from filter material is not probable under normal conditions, the risks associated with handling filters with mycobacteria in ventilation systems under field-use conditions have not been evaluated. Allow employees concerned about changing out the EA filters from the three HRUs to disinfect the used filters with a $10 \%$ bleach solution or other appropriate mycobacteriacide before removal.

\section{Personal Protective Equipment}

Personal protective equipment is the least effective means for controlling hazardous exposures. Proper use of personal protective equipment requires a comprehensive program and a high level of employee involvement and commitment. The right personal protective equipment must be chosen for each hazard. Supporting programs such as training, changeout schedules, and medical assessment may be needed. Personal protective equipment should not be the sole method for controlling hazardous exposures. Rather, personal protective equipment should be used until effective engineering and administrative controls are in place.

1. Ensure standard procedures for PPE are clearly established, communicated to employees, and enforced by supervisors.

2. Ensure employees wear a respirator (N95 or higher), eye protection, and gloves when performing maintenance and replacing exhaust air filters from any ventilation system with potential for M. tuberculosis contamination.

3. Ensure employees wear N95 respirators, disposable coveralls, such as Tyvek, and gloves during any aerosol generating activities, such as power-washing or using highpressure water/cleaning sprays.

4. Ensure employees or volunteers working directly with elephants with TB disease wear respiratory protection (N95 respirator or higher) until the lead veterinarian deems the elephant to be non-infectious.

5. Make N95 disposable filtering-face piece respirators available for voluntary use for protection against tuberculosis when working around any elephant. Elephants in this herd have been exposed to TB and could develop active TB at any time. Because there is a lag time between when an elephant starts shedding TB and the positive trunk wash culture result, workers may be exposed to active TB during that time period. Wearing a N95 respirator would decrease the risk of exposure. N95 respirators should be available in various sizes, and each potential N95 user should receive a copy of 
Appendix D of the OSHA Respiratory Protection Standard (http:/www.osha.gov/pls/ oshaweb/owadisp.show document?p table=standards\&p id=9784). Information about Appendix D and voluntary use of respirators can be found on the OSHA website at https://www.osha.gov/video/respiratory protection/voluntaryuse transcript.html.

6. Ensure employees who work with elephants and in the elephant area participate in the zoo's TB Monitoring Program and Respiratory Protection Program.

7. Ensure employees are trained to properly wear N95 respirators. This should include what to do if N95 respirators becomes compromised or wet. If employees are unable to wear N95 respirators, they become wet during certain procedures, or enhanced comfort is needed due to heat or humidity, powered air-purifying respirators (PAPRs) with loose-fitting facepieces or hoods are an option.

8. Ensure employees are trained and fit-tested annually to wear an N95 respirator and keep records. 


\section{References}

Chalke HD [1962]. The impact of tuberculosis on history, literature and art. Med Hist 6(4):301-318.

Division of Tuberculosis Elimination [2014]. Tuberculosis (tb). https://www.cdc.gov/tb/publications/factsheets/general/ltbiandactivetb.htm: Centers for Disease Control and Prevention.

Feldman M, Isaza R, Prins C, Hernandez J [2013]. Point prevalence and incidence of mycobacterium tuberculosis complex in captive elephants in the united states of america. Vet Q 33(1):25-29.

Jensen PA, Lambert LA, Iademarco MF, Ridzon R, Cdc [2005]. Guidelines for preventing the transmission of mycobacterium tuberculosis in health-care settings, 2005. MMWR Recomm Rep 54(RR-17):1-141.

Ko G, Burge H, Muilenberg M, Rudnick S, First M [1998]. Survival of mycobacteria on hepa filter material Applied Biosafety 3(2):65-78.

Michalak K, Austin C, Diesel S, Bacon MJ, Zimmerman P, Maslow JN [1998].

Mycobacterium tuberculosis infection as a zoonotic disease: Transmission between humans and elephants. Emerg Infect Dis 4(2):283-287.

Occupational Safety and Health Administration Occupational safety and health standards. U.S. Department of Labor. e-Code of Federal Regulations (29 CFR 1910).

Qian Y, Willeke K, Grinshpun SA, Donnelly J [1997]. Performance of N95 respirators: Reaerosolization of bacteria and solid particles. Am Ind Hyg Assoc J 58(12):876-880.

Reponen TA, Wang Z, Willeke K, Grinshpun SA [1999]. Survival of mycobacteria on n95 personal respirators. Infect Control Hosp Epidemiol 20(4):237-241.

Rutala WA, Cole EC, Wannamaker NS, Weber DJ [1991]. Inactivation of mycobacterium tuberculosis and mycobacterium bovis by 14 hospital disinfectants. Am J Med 91(3B):267S$271 \mathrm{~S}$.

Stakeholders Task Force on Management \& Research Priorities of Tuberculosis in Elephants [2017]. 2017 recommendations for the diagnosis, treatment and management of tuberculosis (mycobacterium tuberculosis) in elephants in human care. http://www.aazv.org/.

United States Animal Health Association (USAHA) Elephant Tuberculosis Submcommittee [2012]. Guidelines for the control of tuberculosis in elephants 2012 (draft revision). http:// www.usaha.org/upload/Committee/Tuberculosis/TB\%20Guidelines\%202012\%20Draft\%20 revision\%2020April2012.pdf. 
Zlot A, Vines J, Nystrom L, Lane L, Behm H, Denny J, Finnegan M, Hostetler T, Matthews G, Storms T, DeBess E [2016]. Diagnosis of tuberculosis in three zoo elephants and a human contact - oregon, 2013. MMWR Morb Mortal Wkly Rep 64(52):1398-1402, 10.15585/mmwr. mm6452a2. 
This page left intentionally blank 
Keywords: NAICS 712130 Zoos, live animal exhibits, parks, and wild animals 
The Health Hazard Evaluation Program investigates possible health hazards in the workplace under the authority of the Occupational Safety and Health Act of 1970 (29 U.S.C. § 669(a) (6)). The Health Hazard Evaluation Program also provides, upon request, technical assistance to federal, state, and local agencies to investigate occupational health hazards and to prevent occupational disease or injury. Regulations guiding the Program can be found in Title 42, Code of Federal Regulations, Part 85; Requests for Health Hazard Evaluations (42 CFR Part 85).

\section{Disclaimer}

The recommendations in this report are made on the basis of the findings at the workplace evaluated and may not be applicable to other workplaces.

Mention of any company or product in this report does not constitute endorsement by the National Institute for Occupational Safety and Health (NIOSH).

Citations to Web sites external to NIOSH do not constitute NIOSH endorsement of the sponsoring organizations or their programs or products. NIOSH is not responsible for the content of these Web sites. All Web addresses referenced in this document were accessible as of the publication date.

\section{Acknowledgments}

Desktop Publisher: Tia McClelland

Site Visit Team Members: Suzanne Tomasi and Jonathan Wortham

\section{Availability of Report}

Copies of this report have been sent to the employer, employees, and union at the facility. The state and local health department and the Occupational Safety and Health Administration Regional Office have also received a copy. This report is not copyrighted and may be freely reproduced.

This report is available at http://www.cdc.gov/niosh/hhe/reports/pdfs/2017-0096-3318.pdf.

All other Health Hazard Evaluation Reports may be found at http://www2a.cdc.gov/hhe/ search.asp.

\section{Recommended citation for this report:}

$\mathrm{NIOSH}$ [2018]. Health hazard evaluation report: Evaluation of potential employee exposures to Mycobacterium tuberculosis in a zoo's elephant exhibit. By Reynolds LE, Martin SB, Harvey RR. Morgantown, WV: U.S. Department of Health and Human Services, Centers for Disease Control and Prevention, National Institute for Occupational Safety and Health, NIOSH HHE Report No. 2017-0096-3318. 
Delivering on the Nation's promise:

Safety and health at work for all people through research and prevention

To receive documents or other information about occupational safety and health topics, contact NIOSH

Telephone: 1-800-CDC-INFO (1-800-232-4636)

TTY: 1-888-232-6348

email: cdcinfo@cdc.gov

or visit the NIOSH website at http://www.cdc.gov/niosh

SAFER • HEALTHIER $\cdot$ PEOPLE $^{\text {TM }}$ 